\title{
USE OF EXOPOLYSACCHARIDE - PRODUCING Streptococcus thermophilus IN IMPROVING REDUCED-FAT LABNEH \\ El-Sharoud, W.M. ${ }^{*}$ and K.M. Ayad** \\ * Food Safety and Microbial Physiology Laboratory, Faculty of Agriculture, Mansoura University \\ ** Animal Production Research Institute, Agricultural Research Centre, Ministry of Agriculture, Giza
}

\begin{abstract}
The effect of using exopolysaccharide(EPS)-producing Streptococcus thermophilus on the chemical, textural and sensory characteristics of Labneh (concentrated yoghurt) was studied. Labneh was prepared from full-fat, half-fat and quarter-fat buffalo's milk by using EPS-producing $S$. thermophilus strain and its isogenic EPS-nonproducing mutant culture. The use of EPS-producing starter was generally associated with higher moisture and moisture in non-fat substance (MNFS) contents and higher $\mathrm{pH}$ values in resultant Labneh. EPS also increased water-holding capacity (WHC) of Labneh prepared from full-fat and half-fat buffalo's milk compared with their counterpart products prepared with EPS-nonproducing starter. However, EPS improved the textural and sensory characteristics of only reduced-fat Labneh with insignificant differences $(P<0.05)$ in examined properties between full-fat and half-fat Labneh prepared with EPS-producing and EPS-nonproducing $S$. thermophilus. It was suggested that mechanisms other than the basic ability of EPS to cause water retention may be involved in the improving effect of EPS on textural and sensory characteristics of reduced-fat Labneh. These mechanisms may include the interfering effect of EPS on protein-protein interactions that could soften the curd and improve textural and sensory characteristics.
\end{abstract}

\section{INTRODUCTION}

Due to consumers' awareness of the negative health impact of regular uptake of elevated levels of dietary fat, consumers have developed an interest in low-fat dairy products since the mid to late 1970's (Mistry, 1995). However, growth in the low-fat dairy products market has been slower than would be anticipated on the basis of this consumer inclination (Banks, 2004). This is because of the inferior taste and texture characteristics caused by lowering fat content. Microscopic examinations of full-fat and reduced-fat Mozzarella cheese types showed that much of the water in cheese is contained in channels formed in the protein matrix by entrapped fat globules (Oberg et al., 1993). These channels are diminished and thus retain less water in low-fat cheese because of the decrease in the fat globules that would break up the protein matrix. This leads to the production of cheese of lower moisture in the non-fat substance (MNFS) content, which is associated with a tough, rubbery texture and poor melt and stretch properties (McMahon \& Oberg 1998). There have been a number of approaches adopted to help overcome quality defects of low-fat dairy products and these approaches included the control of processing variables, e.g. milk type, application of 
homogenization, the selection of appropriate starter and adjunct bacteria, and the use of fat mimetics to improve texture (Banks, 2004). Exopolysaccharides produced by lactic acid bacteria have been shown to be an appropriate means for improving the quality of low-fat cheese because of their excellent water-binding properties (Broadbent et al., 2003). However, this effect was only examined in certain types of cheeses including Mozzarella cheese (Perry et al., 1997; Low et al., 1998) and Cheddar cheese (Awad et al., 2005) with almost no further data available on other dairy products.

Labneh is a concentrated/strained fermented milk that is widely consumed in the Middle East and Balkan regions (Tamime et al., 1989; Ozer et al., 1999; Al-Kadamany et al., 2002; Nsabimana et al., 2005). Compared with other yoghurt types, Labneh and Greek Style yoghurt are very rich in fat (Tamime \& Robinson 2000), which makes its regular consumption posing a significant health risk. Producing reduced-fat Labneh of acceptable characteristics is therefore needed to keep it as beloved and healthy food. In this work, we examined the possibility of improving the quality of reduced-fat Labneh prepared from buffalo's milk by using exopolysaccharide (EPS)producing Streptococcus thermophilus. We show that it is possible to improve the texture and sensory qualities of reduced-fat Labneh by using EPS-producing $S$. thermophilus, but this appears not to depend on the basic ability of EPS to cause water retention in the curd.

\section{MATERIALS AND METHODS}

\section{LAB Cultures and Growth Conditions}

Three strains of Streptococcus thermophilus were used in this study. These included the CNRZ1066 strain which is EPS ${ }^{+}$, the JIM8752 strain, which is an isogenic mutant of CNRZ1066 that does not synthesize EPS and the FSMP111 strain, which is a natural strain isolated from dairy products and does not produce EPS. Strains CNRZ1066 and JIM8752 were generously gifted by Dr. Pierre Renault of the Genetique Microbienne, INRA-CRJ, France, but strain FSMP111 was isolated from yoghurt by staff members of the food safety and microbial physiology (FSMP) laboratory at Mansoura University, Egypt. Cultures were propagated in sterilized reconstituted skim milk ( $10 \%$ total solids) at $37^{\circ} \mathrm{C}$ prior to use in the preparation of Labneh.

\section{Preparation of Labneh}

Full-fat $(7 \%)$, half-fat $(3.5 \%)$ and quarter-fat (1.75\%) buffalos' milk was pasteurized, warmed to $35^{\circ} \mathrm{C}$ and divided into aliquots, that were inoculated with $2 \%$ of $S$. thermophilus CNRZ1066 (EPS + ), FSMP111(EPS+) or JIM8752 (EPS $)$. Milk was then adjusted to $30^{\circ} \mathrm{C}$ and incubated for $24 \mathrm{~h}$ for coagulation. These incubation conditions were adopted since they were previously shown to enable the optimum production of EPS by $S$. thermophilus (Mozzi et al., 1995; Hassan 2008). Resultant curd was then slowly stirred and packed into cloth bags that were stacked on top of each other and left for 1-2 days under refrigeration. On obtaining appropriate consistency, concentrated curd were removed from the bags, blended with 1 $2 \%$ salt, distributed into plastic containers and kept refrigerated for 2 weeks. 


\section{Chemical Analysis and pH Determination}

Moisture and fat contents of Labneh were analysed using the AOAC standard methods (AOAC, 2003). $\mathrm{pH}$ was measured using Corning $240 \mathrm{pH}$ meter (Corning, Suffolk, UK).

\section{Measurement of Water-holding Capacity}

Water-holding capacity (WHC) of Labneh samples were estimated using the method described by Parnell-Clunies et al., (1986). Samples were centrifuged at $13,500 \times g$ for $30 \mathrm{~min}$ at $10^{\circ} \mathrm{C}$. Supernatant was discarded and pellet were left to drain for $15 \mathrm{~min}$. WHC was expressed as the percentage of dry pellet weight relative to the original weight of the sample.

\section{Texture Analysis}

The following textural properties were determined: a) hardness (force required to attain a given deformation), b) chewiness (energy required to chew a food product to a state where it is ready for swallowing, c) consistency (work required to attain deformation indicative of internal strength of bonds within product), d) load of relaxation (samples are rapidly deformed to a pre-determined extent and the ensuing stress is measured as a function of time at a constant deformation), e) cohesiveness (the strength of internal bonds making up the body of the product) (Szczesniak et al. 1963). Textural properties of Labneh samples were assessed using texture analyzer (CNSFarnell, Borehamwoad, Hertfordshimre, England). Examined samples were allowed to equilibrate at ambient temperature for approximately 30-45 min before testing and were then presented to the instruments. The examined samples were $30 \mathrm{~mm}$ in diameter and $20 \mathrm{~mm}$ in height. A TA15-451 Perspex cone was used as the probe with a penetration of $10 \mathrm{~mm}$ at $1 \mathrm{~mm} / \mathrm{s}$. Textural characteristics were calculated from the LFRA Texture Analyser $(1000 \mathrm{~g})$ using Computer Interface Software (CNS Farnell, Borehamwoad, Hertfordshimre, England).

\section{Sensory Evaluation}

Labneh samples were cut into pieces of appropriate sizes and placed on white plates. Samples were tempered at ambient temperature and then presented to 15 panelists in a random order. Panelists evaluated Labneh for appearance, body \& texture and flavor. Scores were obtained for the three sensory attributes and panelists were also asked to report detailed comments on their preference towards the examined samples.

\section{Sampling and Experiment Replication}

In all the above examinations, samples were taken immediately after the preparation of Labneh and weekly for 2 weeks of cold storage. Each experiment was carried out 3 times.

\section{Statistical Analysis}

All presented data are the means of at least 3 replicates. Means were statistically compared according to the LSD test and differences were considered significant at $\mathrm{P}<0.05$ (Little \& Hills, 1996). 


\section{RESULTS AND DISCUSSION}

\section{Manufacture and Chemical Composition of Labneh:}

Labneh was prepared from full-fat, half-fat and quarter-fat buffalo's milk with the use of $S$. thermophilus JIM8752 (EPS-) (treatments $1,2,3$ ) or $S$. thermophilus CNRZ1066 (EPS ${ }^{+}$) (treatments 4, 5, 6). The fat content of the resultant Labneh products ranged from 6.6 to 22.0 (table 1). The decrease in fat content in buffalo's milk used for preparing Labneh was associated with an increase in moisture content (table 1). This was noticed whether with the use of EPS-non producing $S$. thermophilus (treatments $1,2,3$ ) or with EPSproducing $S$. thermophilus (treatments $4,5,6)$. However, Labneh samples in the latter treatments showed higher moisture and moisture in non-fat substance (MNFS) contents compared with their counterparts in treatments $1,2,3$. This suggests that the use of EPS-producing $S$. thermophilus increased water retention in Labneh, which is consistent with Petersen et al. (2000) and Low et al. (1998) who reported increased water retention in Mozzarella cheese prepared with EPS-producing S. thermophilus.

Decreasing fat content was associated with decreases in $\mathrm{pH}$ of resultant Labneh in both EPS-nonproducing treatments $(1,2,3)$ and EPSproducing treatments $(4,5,6)$ (table 1$)$. This could be attributed to the concomitant increases in moisture content, which decrease the concentrations of hydrogen ions in the resultant Labneh. However, it is noticed that higher $\mathrm{pH}$ values were recorded for Labneh prepared with EPSproducing $S$. thermophilus (treatments $4,5,6)$ compared with their corresponding treatments $(1,2,3)$, in which EPS nonproducing $S$. thermophilus was used. Here too, this may be a reflection of relatively higher water retention rates associated with the use of EPS-producing $S$. thermophilus that lower the concentrations of hydrogen ions in resultant Labneh. However, Hassan et al. (1995) reported that encapsulated strains of $S$. thermophilus produced less acid in milk than did their unencapsulated counterpart strains. Two explanations have been suggested for this phenomenon.

Table 1: Effect of using EPS-producing S. thermophilus on the chemical composition and $\mathrm{pH}$ of Labneh*

\begin{tabular}{|c|c|c|c|c|}
\hline Treatment & F\% & M\% & MNFS\% & $\mathbf{p H}$ \\
\hline $\mathbf{1}$ & $22.00 \pm 0.15$ & $66.75 \pm 0.42$ & 85.58 & $4.36 \pm 0.03$ \\
\hline $\mathbf{2}$ & $13.63 \pm 0.10$ & $71.00 \pm 0.54$ & 82.20 & $4.51 \pm 0.02$ \\
\hline $\mathbf{3}$ & $6.60 \pm 0.06$ & $83.25 \pm 0.53$ & 89.13 & $4.74 \pm 0.01$ \\
\hline $\mathbf{4}$ & $22.03 \pm 0.03$ & $68.00 \pm 0.43$ & 87.21 & $4.76 \pm 0.03$ \\
\hline $\mathbf{5}$ & $13.60 \pm 0.04$ & $72.50 \pm 0.48$ & 83.91 & $4.98 \pm 0.04$ \\
\hline $\mathbf{6}$ & $6.64 \pm 0.08$ & $85.25 \pm 0.44$ & 91.31 & $5.03 \pm 0.01$ \\
\hline
\end{tabular}

* Presented values are the means of 3 replicates $\pm \mathrm{SE}$.

Treatment 1: Full-fat Labneh + JIM8752

Treatment 2: Half-fat Labneh + JIM8752

Treatment 3: Quater-fat Labneh + JIM8752

Treatment 4: Full-fat Labneh + CNRZ1066

Treatment 5: Half-fat Labneh + CNRZ1066

Treatment 6: Quarter-fat Labneh + CNRZ1066 
Firstly, it was proposed that EPS-producing $S$. thermophilus may spend more energy to produce capsules and therefore grow more slowly and produce less acid. Secondly, the formation of EPS-capsules around the cells may cause acid accumulation in the cytoplasm that inhibits metabolic activities leading to acid production.

Similar patterns of chemical composition and $\mathrm{pH}$ were recorded during cold storage of Labneh, where slight changes in the examined chemical attributes and $\mathrm{pH}$ were found.

\section{Effect of fat reduction and EPS on Water-Holding Capacity:}

Water-holding capacity (WHC) of a dairy product is a measure of the ability of its matrix to bind water or hold entrapped or bulk phase water (Parnell-Clunies et al., 1986). Reducing fat in Labneh prepared with EPSnonproducing or EPS-producing $S$. thermophilus was associated with decreases in WHC in the resultant product and this was more pronounced in Labneh prepared with EPS-producing $S$. thermophilus (Figure 1). This confirms previous reports showing the role of fat in water retention in Mozerall cheese (Oberg et al., 1993; McMahon \& Oberg 1998; Broadbent et al., 2003) and Cheddar cheese (Hassan \& Awad, 2005.). It was suggested in these studies that fat globules form channels in the protein matrix, within which water is entrapped and the reduction of fat content in dairy products could thus reduce these channels and cause less water retention.

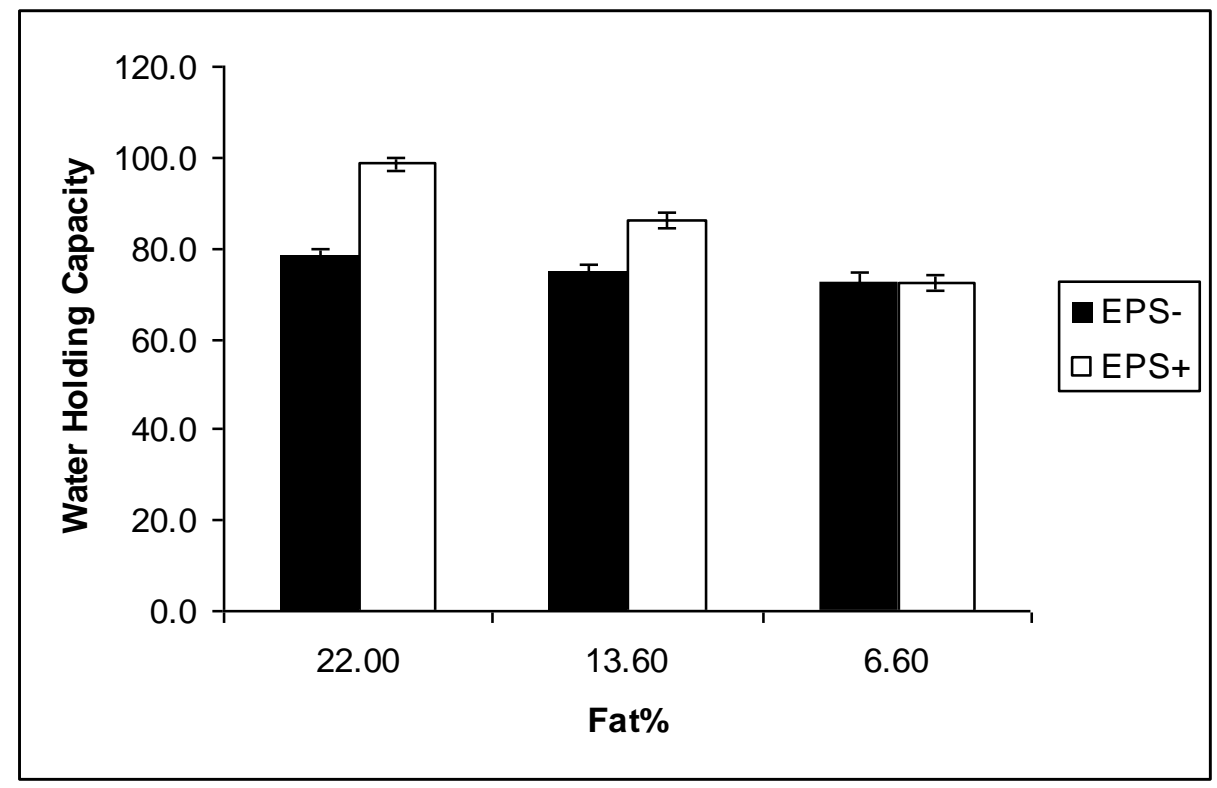

Figure 1: Water-holding capacity of Labneh prepared by the EPSnonproducing $S$. thermophilus JIM8752 strain ( $\square$ ) and EPSproducing $S$. thermophilus CNRZ1066 strain ( $\square$ ). Values represent the means of 3 replicates $\pm S E$. 
It could be noticed in figure 1 that higher WHC values were recorded in full-fat $(22.00 \%)$ and half-fat $(13.60 \%)$ Labneh prepared by using EPSproducing $S$. thermophilus than that prepared with its EPS-nonproducing isogenic mutant strain. No such a difference was found between quarter-fat Labneh $(6.60 \%)$ treatments prepared by EPS-producing and EPSnonproducing strains. This suggests that EPS increases the ability of the acid curd of Labneh to bind water or hold entrapped water, but this depends on the fat content of the product. These results are consistent with previous reports showing that the use of encapsulated LAB starter in the preparation of full-fat yoghurt increased the WHC of the final product (Hassan et al., 1996), but no difference in WHC could be detected between nonfat yogurt fermented by EPS-producing and EPS-nonproducing starters (Schellhaass \& Moris 1985).

\section{Effect of fat reduction and EPS on texture properties of Labneh:}

Texture properties of full-fat, half-fat and quarter-fat Labneh prepared by using EPS-nonproducing and EPS-producing $S$. thermophilus were assessed in fresh product and after 1 and 2 weeks of cold storage. Examined properties included hardness, consistency, chewiness, load of relaxation and cohesiveness. Figures $2 \mathrm{a}$ through $2 \mathrm{e}$ show the results of fresh Labneh, from which two general patterns could be deduced. Firstly, reducing fat content was associated with changes in the values of examined texture characteristics in Labneh prepared by EPS-nonproducing and EPS-producing starters. While these changes were slight and insignificant $(P<0.05)$ in Labneh prepared by EPS-nonproducing $S$. thermophilus, they were significant $(P<0.05)$ in Labneh produced by EPS-producing starter. Secondly, significant differences in textural properties $(P<0.05)$ could be only detected between reduced-fat Labneh treatments $(6.60 \% \mathrm{~F})$ prepared by EPS-nonproducing and EPS-producing $S$. thermophilus, with insignificant differences $(P<0.05)$ in these properties between full-fat $(22.00 \% \mathrm{~F})$ and half-fat $(13.60 \% \mathrm{~F})$ treatments. These patterns remained without change during cold storage of Labneh, where there were only slight changes in the values of examined properties (data not shown).

The use of EPS-producing $S$. thermophilus in the preparation of Labneh was associated with significantly lower values $(P<0.05)$ of hardness, chewiness, consistency and load of relaxation (Figures 2a-2d) and significantly higher values $(P<0.05)$ of cohesiveness in reduced-fat Labneh $(6 \% \mathrm{~F})$ compared with its counterpart prepared with non-producing $S$. thermophilus (Figure 2e). These results are in agreement with those of Ahmed etal.(2005) and Hassan etal. (2005) who reported similar findings with the low-fat Kariesh cheese and reduced-fat Cheddar cheese, respectively. These differences in textural properties could be attributed to the higher water retention indicated by higher moisture and MNFS contents of reduced-fat Labneh prepared with EPSproducing S. thermophilus (treatment 6 ) compared with its corresponding treatment (treatment 3 ), in which EPS-nonproducing starter was used (table 1). Beal and Mittal (2000) suggested that water weakens the protein network and thus softens curd, lowering hardness, chewiness, consistency and load of relaxation and increasing cohesiveness. However, it could be seen in table 1 that moisture content and MNFS content were generally higher in Labneh 
prepared with EPS-producing starter compared with its EPS-nonproducing counterparts prepared from full-fat or half-half milk. It could be also seen in figure 1 that WHC values were higher in full-fat and half-fat Labneh prepared with EPS-producing starter than those of Labneh containing the same fat contents but made with EPS nonproducing $S$. thermphilus. Interestingly, there was no difference in WHC between reduced-fat Labneh prepared with EPSproducing and EPS-nonproducing S. thermophilus.

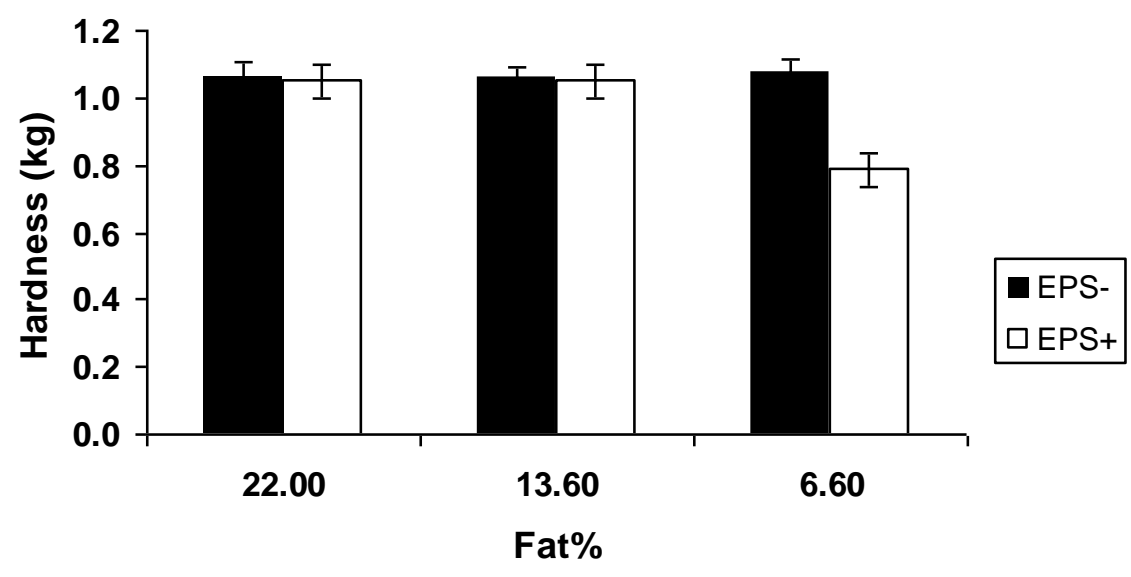

Figure 2a:_ Effect of using EPS-nonproducing S. thermophilus JIM8752 strain (घ) and EPS-producing S. thermophilus CNRZ1066 strain (口) on the hardness of Labneh. Values represent the means of 3 replicates $\pm S E$.

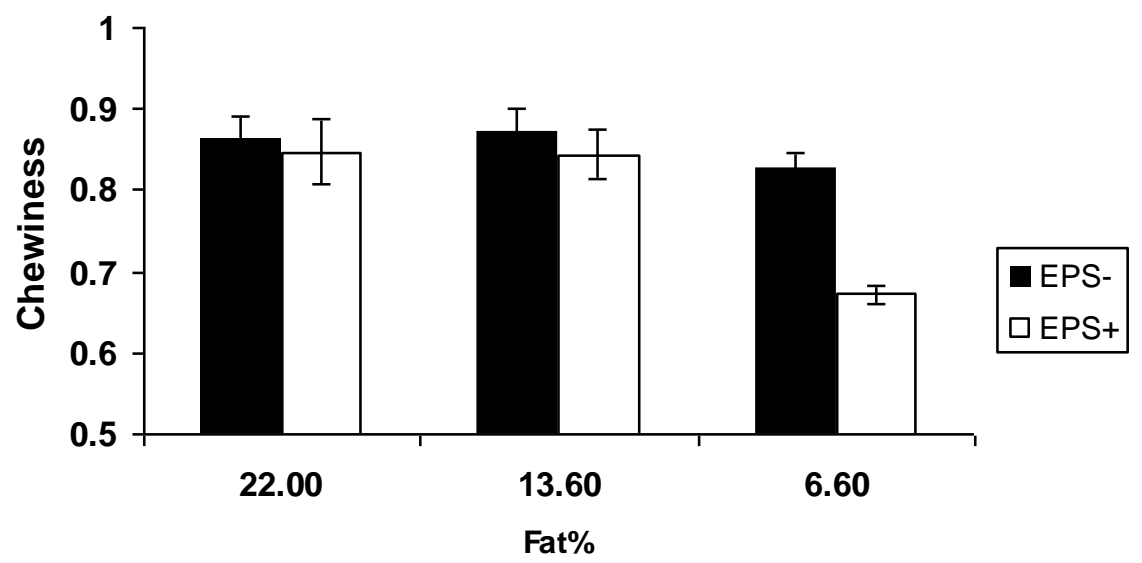

Figure 2b: Effect of using EPS-nonproducing S. thermophilus JIM8752 strain (a) and EPS-producing S. thermophilus CNRZ1066 strain ( $\square$ ) on the chewiness of Labneh. Values represent the means of 3 replicates \pm SE. 


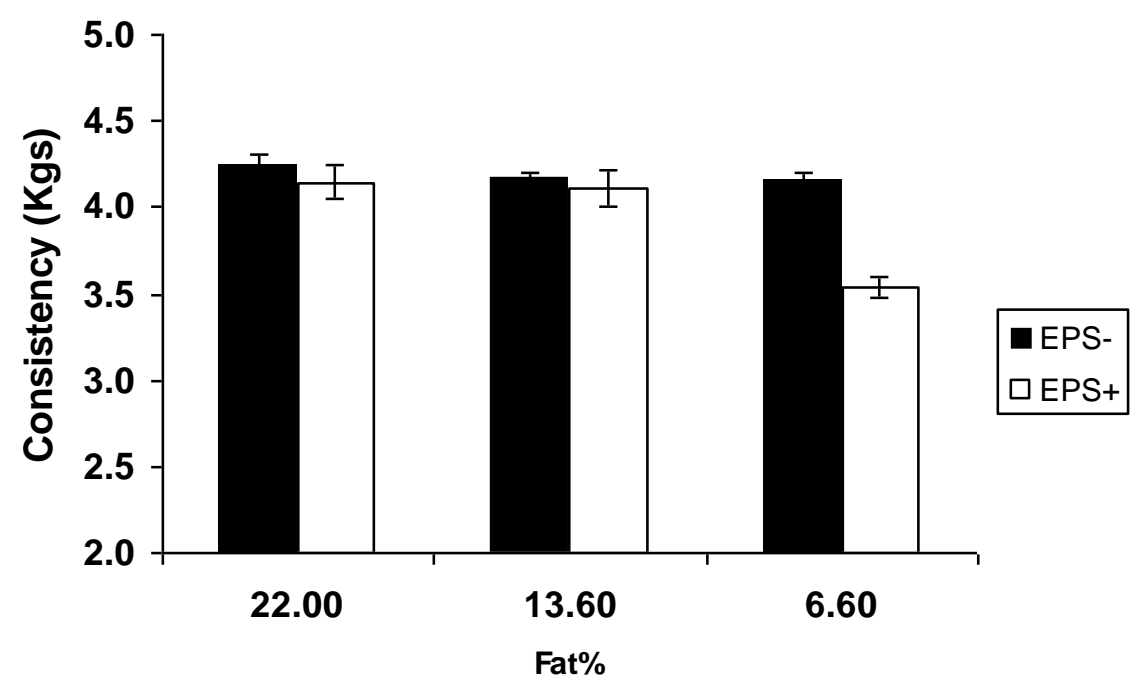

Figure 2c: Effect of using EPS-nonproducing S. thermophilus JIM8752 strain ( $\square$ ) and EPS-producing S. thermophilus CNRZ1066 strain ( $\square$ ) on the consistency of Labneh. Values represent the means of 3 replicates \pm SE.

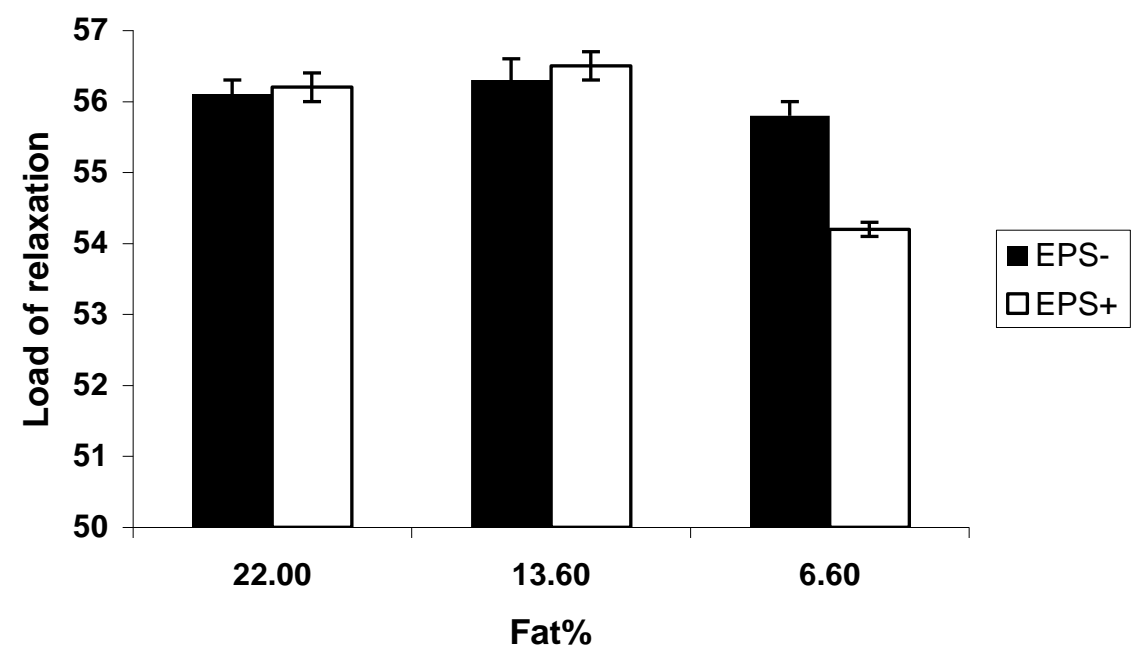

Figure 2d: Effect of using EPS-nonproducing S. thermophilus JIM8752 strain (a) and EPS-producing S. thermophilus CNRZ1066 strain (口) on the load of relaxation of Labneh. Values represent the means of 3 replicates \pm SE. 


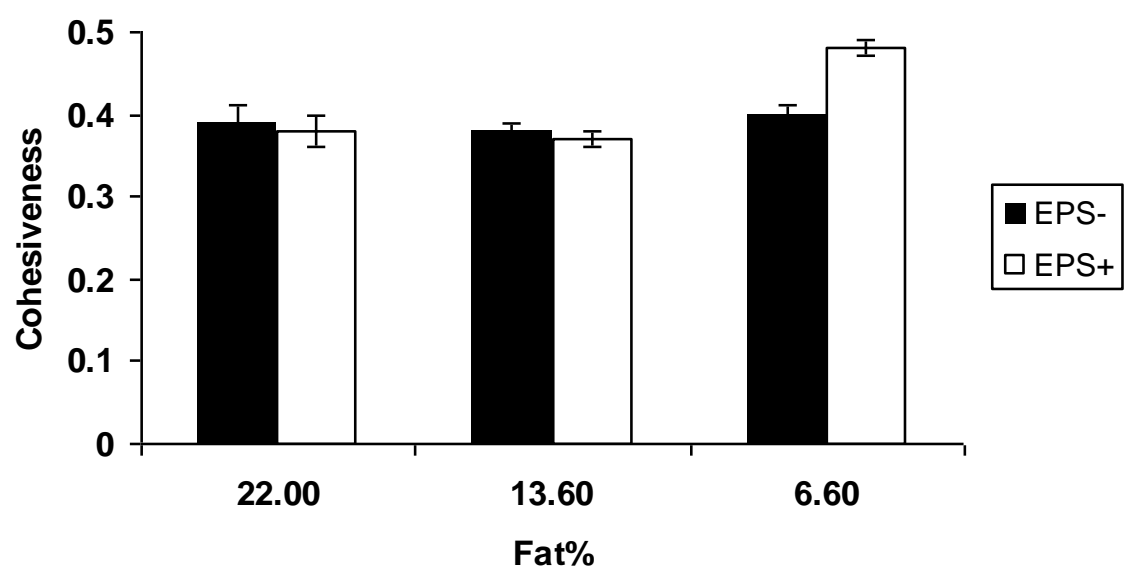

Figure 2e: Effect of using EPS-nonproducing S. thermophilus JIM8752 strain (a) and EPS-producing S. thermophilus CNRZ1066 strain ( $\square$ ) on the cohesiveness of Labneh. Values represent the means of 3 replicates \pm SE.

Together, these findings suggest that the effect of EPS on textural properties of Labneh is dependent on fat content of the product and this effect is only pronounced in reduced-fat Labneh. It could be also concluded that there are other mechanisms of the influence of EPS on Labneh beside their basic ability to cause water retention within the curd. These mechanisms may involve an interfering effect of EPS on protein-protein interactions that could soften the curd (Hassan \& Frank, 1997; Hassan et al., 2003). It was reported that EPS could disrupt the continuity of the protein network in which the protein-protein bonds are contributing to the rigidity of curd (Green et al. 1978). This leads to a less tighter network structure in the resultant product. Based on our current results, the significance of the interfering effect of EPS on protein-protein interactions on the texture of Labneh is not noticed with high fat content $(22.0 \%$ or $13.60 \%)$ but in reduced-fat product $(6.0 \%)$.

\section{Sensory evaluation of Labneh}

Sensory characteristics (appearance, body \& texture, and flavour) of Labneh prepared with EPS-producing S. thermophilus CNRZ1066 were compared with those of Labneh prepared with non-producing $S$. thermophilus FSMP111. The isogenic mutant EPS-nonproducing S. thermophilus JIM8752 strain was not used in this part of the study given safety precautions over carrying out taste evaluation of foods containing genetically modified (GM) organisms. There were no significant differences $(P<0.05)$ in appearance between Labneh produced by EPS-nonproducing starter and that prepared with EPS-producing S. thermophilus at different fat levels (Table 2). However, significantly higher scores were assigned to the body \& texture of reduced-fat Labneh prepared with EPS-producing $S$. thermophilus (treatment 6) 
compared with its counterpart made with EPS-nonproducing strain (treatment $3)$. There were no significant differences $(P<0.05)$ in body \& texture between the full-fat and half-fat Labneh prepared with EPS-nonproducing starter (treatments 1 and 2, respectively) and their counterparts prepared with EPSproducing starter (treatments 4 and 5 , respectively). These patterns were also noticed with the flavour, where significant differences $(P<0.05)$ were only noticed between reduced-fat Labneh prepared by EPS-producing starter (treatment 6) and its counterpart prepared with EPS-nonproducing starter (treatment 3). Interestingly, panellists favoured the body and texture and flavour of reduced-fat Labneh prepared by EPS-producing $S$. thermophilus (treatment 6) over full-fat Labneh prepared with EPS-nonproducing and EPSproducing organisms (treatments 1 and 4, respectively). Panellists provided comments that the latter treatments had rigid body and gave unacceptable fatty taste. Whereas, reduced fat Labneh prepared with EPS-producing $S$. thermophilus had preferred soft body, smooth texture and fat-rich mouthfeeling.

Table 2: Effect of using EPS-producing S. thermophilus on the sensory evaluation of Labneh*

\begin{tabular}{|c|c|c|c|}
\hline \multirow{2}{*}{ Treatment } & \multicolumn{3}{|c|}{ Property } \\
\cline { 2 - 4 } & Appearance (15) & Body \& Texture (35) & Flavor (50) \\
\hline $\mathbf{1}$ & 13 & 30 & 42 \\
\hline $\mathbf{2}$ & 12 & 28 & 42 \\
\hline $\mathbf{3}$ & 12 & 27 & 41 \\
\hline $\mathbf{4}$ & 13 & 31 & 43 \\
\hline $\mathbf{5}$ & 13 & 28 & 44 \\
\hline $\mathbf{6}$ & 13 & 35 & 47 \\
\hline
\end{tabular}

* Presented values are the means of 3 replicates \pm SE.

Treatment 1: Full-fat Labneh + FSMP111

Treatment 2: Half-fat Labneh + FSMP111

Treatment 3: Quater-fat Labneh + FSMP111

Treatment 4: Full-fat Labneh + CNRZ1066

Treatment 5: Half-fat Labneh + CNRZ1066

Treatment 6: Quarter-fat Labneh + CNRZ1066

\section{Conclusions:}

This study reports the possibility of improving the textural and sensory characteristics of reduced-fat Labneh by the use of exopolysaccharideproducing Streptococcus thermophilus. This highlights the value of EPSproducing $L A B$ in overcoming quality defects associated with reducing fat content in dairy products, which paves the way for developing and marketing reduced-fat dairy products with improved quality traits. The study also confirms the ability of EPS to induce water retention in dairy products, but interestingly indicates that this was not the mechanism by which EPS improved the quality of reduced-fat Labneh. A potential mechanism could be the interfering effect of EPS on protein-protein interactions that leads to a less tighter network structure in the resultant product. However, this hypothesis remains to be experimentally addressed within future studies. 


\section{Acknowledgments}

This work was supported by Research Grant No. 060365 awarded to WM El-Sharoud by the Library of Alexandria (BA/CSSP programme 20062007), Alexandria.

\section{REFERENCES}

Ahmed, N.H., El-Soda, M., Hassan, A.N. and Frank, J. (2005). Improving the texture properties of an acid-coagulated (Karish) cheese using exopolysaccharide producing culture. LWT, 38, 843-847.

Al-Kadamany, E., Toufeili, I., Khattar, M., Abou-Jawdeh, Y., Harakeh, S. and Haddad, T. (2002). Determination of shelf life of concentrated yogurt (Labneh) produced by in-bag straining of set yogurt using hazard analysis. Journal of Dairy Science, 85, 1023-1030

Association of Official Analytical Chemists (2003). Official Methods of Analysis. $17^{\text {th }}$ ed. AOAC, Gaithersburg, MD.

Awad, S., Hassan, A.N. and Halaweish, F. (2005). Application of exopolysaccharide-producing cultures in reduced-fat Cheddar cheese: composition and proteolysis. Journal of Dairy Science, 88, 4195-4203.

Banks, J.M. (2004). The technology of low-fat cheese manufacture. International Journal of Dairy Technology, 57, 199-208.

Beal, p., and Mittal, G.S. (2000). Vibration and Compresion responses of cheddar cheese at different fat content and age. Milchwissenschaft, 55 , 139-142.

Broadbent, J.R., McMahon, D.J., Welker, D.L., Oberg, C.J. and Moineau, S. (2003). Biochemistry, genetics and applications of exopolysaccharide production in Streptococcus thermophilus. Journal of Dairy Science, $86,407-423$.

Green, M.L., Hobbs, D.G., Morant, S.V. and Hill, V.A. (1978). Intermicellar relationships in rennet-treated separated milk. II. Process of gel assembly. Journal of Dairy Research, 45, 413-422.

Hassan, A.N. (2008). ADSA Foundation Scholar Award: Possibilities and Challenges of Exopolysaccharide-Producing Lactic Cultures in Dairy Foods. Journal of Dairy Science, 91, 1282 - 1298.

Hassan, A.N. and Awad, S. (2005). Application of exopolysaccharideproducing cultures in reduced-fat Cheddar cheese: cryo-scanning electron microscopy observations. Journal of Dairy Science, 88, 42144220.

Hassan, A.N. and Frank, J.F. (1997). Modification of microstructure and texture of rennet curd by using a capsule-forming non-ropy lactic culture. Journal of Dairy Research, 64, 115-121

Hassan, A.N., Awad, S., Muthukumarappan, K. (2005). Effects of exopolysaccharide-producing cultures on the viscoelastic properties of reduced-fat Cheddar cheese. Journal of Dairy Science, 88, 4221-4227.

Hassan, A.N., Frank, J.F. and Elsoda, M. (2003). Observation of bacterial EPS in dairy products using cryo-scanning electron microscopy. International Dairy Journal, 13, 755-762. 
Hassan, A.N., Frank, J.F., Farmer, M.A., Schmidt, K.A. and Shalabi, S.I. (1995). Observation of encapsulated lactic acid bacteria using confocal scanning laser microscopy. Journal of Dairy Science, 78, 2624-2628.

Hassan, A.N., Frank, J.F., Schmidt, K.A. and Shalabi, S.I. (1996). Textural properties of yogurt made with encapsulated nonropy lactic cultures. Journal of Dairy Science, 79, 2091-2103.

Little, T. M., and Hills, F.J. (1996). Agriculture experimentation. Design and Analysis, Lengman Gr. Ltd.

Low, D., Ahlgren, J.A., Horne, D., McMahon, D.J., Oberg, C.J. and Broadbent, J.R. (1998). Role of Streptococcus thermophilus MR-1C capsular exopolysaccharide on cheese moisture retention. Applied and Environmental Microbiology, 64, 2147-2151.

McMahon, D.J. and Oberg, C.J. (1998). Influence of fat, moisture, and salt on functional properties of Mozzarella cheese. Australian Journal of Dairy Technology, 53, 98-101.

Mistry, V.V. (1995). Improving the sensory characteristics of reduced-fat cheese. lin Chemistry of Structure-Function Relationships in Cheese, eds. E.L. Malin and M.H. Tunick, Plenum Press, New York, pp. 371382.

Mozzi, F., Oliver, G., de Giori, G.S. and de Valdez, G.F. (1995). Influence of temperature on the production of exopolysaccharides by thermophilic lactic acid bacteria. Milchwissenschaft, 50, 80-82.

Nsabimana, C., Jiang, B. and Kossah, R. (2005). Manufacturing, properties and shelf life of labneh: a review. International Journal of Dairy Technology, 58, 129-137

Oberg, C.J., McManus, W.R. and McMahon, D.J. (1993). Microstructure of Mozzarella cheese during manufacture. Food Structure, 12, 251-258.

Ozer, B.H., Stenning, R.A., Grandison, A.S. and Robinson, R.K. (1999). Rheology and Microstructure of Labneh (Concentrated Yogurt). Journal of Dairy Science, 82, 682-689.

Parnell-Clunies, E., Kakuda, Y., Mullen, K., Arnott, D.R. and deMan, J.M.. (1986). Physical properties of yogurt: a comparison of vat versus continuous heating systems of milk. Journal of Dairy Science, 69, 2593-2598.

Perry, D.B., McMahon, D.J. and Oberg, C.J. (1997). Effect of exopolysaccharide-producing cultures on moisture retention in low-fat Mozzarella cheese. Journal of Dairy Science, 80,799-805.

Petersen, B.L., Dave, R.I., McMahon, D.J., Oberg, C.J. and Broadbent, J.R. (2000). Influence of capsular and ropy exopolysaccharide-producing Streptococcus thermophilus on Mozzarella cheese and cheese whey. Journal of Dairy Science, 83, 1952-1956.

Schellhaass, S.M., and Morris, H. (1985). Rheological and scanning electron microscopic examinations of skim milk gels obtained by fermenting with ropy and non-ropy strains of lactic acid bacteria. Food Microstructure, 4, 279-283. 
Szczesniak, A., Brandt, M. and Freidman, H.. (1963). Development of standard rating scales for mechanical parameters and correlation between the objective and sensory texture measurements. Food Technology 22:50-54.

Tamime, A. Y., and Robinson, R.K. (2000). Yoghurt Science and Technology. 2nd ed. CRC Press, Boca Raton, FL.

Tamime, A.Y., Davies, G., Chehade, A.S., and Mahdi, H.A.. (1989). The production of 'labneh' by ultrafiltration: A new technology. Journal of the Society for Dairy Technology, 42, 35-39.

\section{استخدام بكتريا الاستربتوكوكس ثرموفيلس المنتجة للسكريات الخارجية العديدة في

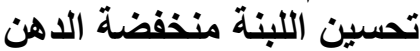

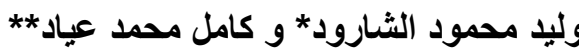

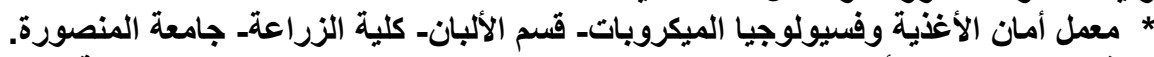

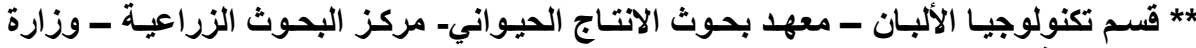

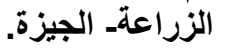

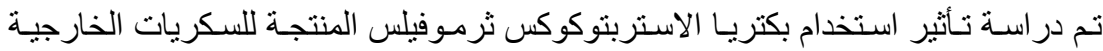

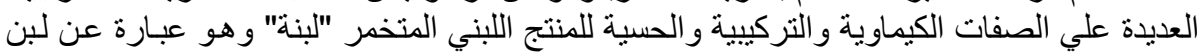

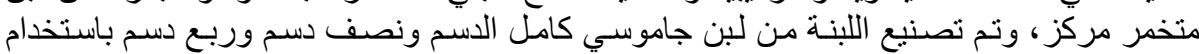

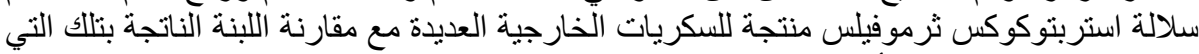

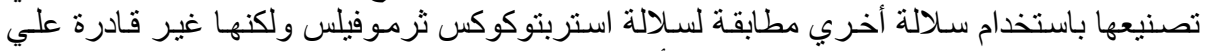

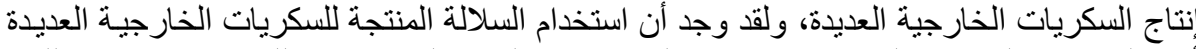



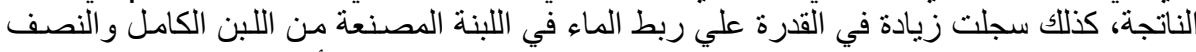

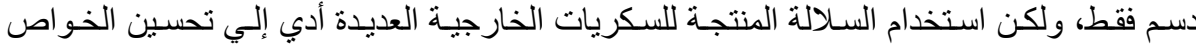

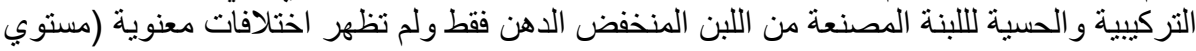

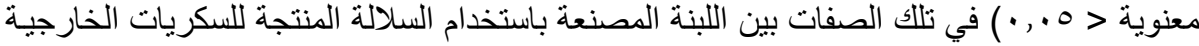

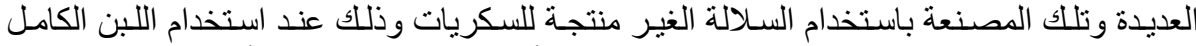

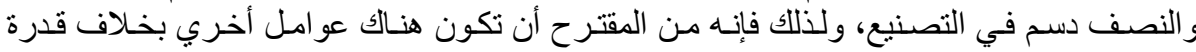

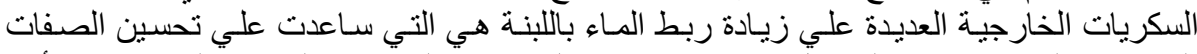

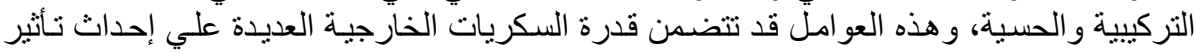

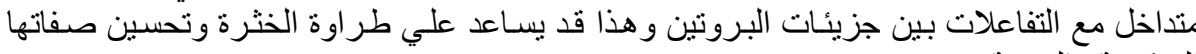
التركيبية والحسية. 